\title{
Development of an open source multi-platform software tool for parameter estimation studies in FMI models
}

\author{
Javier Bonilla $^{1,3}$ Jose A. Carballo ${ }^{1,3}$ Lidia Roca $^{1,3} \quad$ Manuel Berenguel $^{2,3}$ \\ ${ }^{1}$ CIEMAT-PSA, Centro de Investigaciones Energéticas, Medioambientales y Tecnológicas - Plataforma Solar de \\ Almería, Spain, \{javier.bonilla, jose.carballo, lidia.roca\}@psa.es \\ ${ }^{2}$ Department of Informatics, University of Almería, Almería, Spain, beren@ual .es \\ ${ }^{3}$ CIESOL, Solar Energy Research Center, Joint Institute University of Almería - CIEMAT, Almería, Spain
}

\begin{abstract}
This paper presents the current development of an open source multi-platform software tool intended for estimating or optimizing parameters of Functional Mock-up Interface (FMI) compliant models. Parameter estimation and optimization is a powerful tool in many engineering and science fields. Nevertheless, the effort and time that must be devoted to coupling and integrating complex modeling languages and tools together with analysis and optimization methods and algorithms sometimes is high. As a consequence of that, commonly the most convenient and easy-to-use optimization mechanisms are applied. Therefore, the focus on the development of this tool is in facilitating such coupling while being customizable. The main toolkit and libraries used in the development of the tool are presented, all of them are open source. Two application examples are also presented, one of them is a parameter optimization study considering a steady state model, while the other is a parameter estimation study of a dynamic model against experimental data. Finally, current tool limitations are presented, ongoing work and ideas for future features are also commented.
\end{abstract}

Keywords: parameter estimation, parameter optimization, model calibration, Functional Mock-up Interface (FMI), open source software tool

\section{Introduction}

The application of optimization to complex dynamic models has become recently more usual in industry, as well as in academia. Optimization can be online, such as optimal control in the form of Model Predictive Control (MPC) or offline, such as parameter estimation, state estimation or parameter optimization.

In parameter optimization, also known as design optimization, some parameters are optimized to improve the system dynamics or response according to some criteria. Parameter estimation, model calibration or parameter identification, comprises estimating some unknown parameters in a particular model. To that end, several simulations are performed and results are compared against experimental data. The unknown parameters are therefore determined by numerical optimization algorithms. This procedure is a powerful tool in many engineering and science fields and has its origin in the least squares method proposed by Gauss.

In order to apply optimization techniques to complex dynamic models, a suitable modeling language, that can deal with dynamic systems and the increasing complexity of research and engineering needs, is advisable. Modelica (Modelica Association, 2014b) is one of those modeling languages, easing the model development, maintenance and reuse thanks to the equation-based object-oriented paradigm and other useful features. Nevertheless, there are other commonly used modeling languages and simulation tools, being one of the most representative Matlab/Simulink (The MathWorks Inc., 2016). For this reason, the support of an independent standard devoted to Model Exchange (ME) and co-simulation, such as FMI (Modelica Association, 2014a), would be advisable when considering the model interface for an optimization software tool.

Even though there are modeling languages and tools for developing complex dynamic models, as well as a standard format to exchange those models, and advanced methods for optimization, sometimes the time required to couple all these tools is high. This task involves writing scripts for bindings and using several programming languages and tools.

With the aim of facilitating the integration of these tools and methods, an easy-to-use open source multi-platform software tool which performs parameter estimation studies in Functional Mock-up Units (FMUs) is currently being developed. This software tool performs parameter estimation studies using a global-search Multi-Objective Genetic Algorithm (MOGA). The tool also supports linear and non-linear equality and inequality constraints.

This paper is organized as follows. Section 1.1 is a brief summary of Modelica and FMI-based tools for parameter estimation. Section 2 describes the still under development software tool and its architecture. In Section 3, two examples are presented. Section 3.1 shows a steadystate parameter optimization study, whereas Section 3.2 presents a model calibration study against experimental data from a Thermal Energy Storage (TES) tank. This tank is used for research on solar thermal storage. 


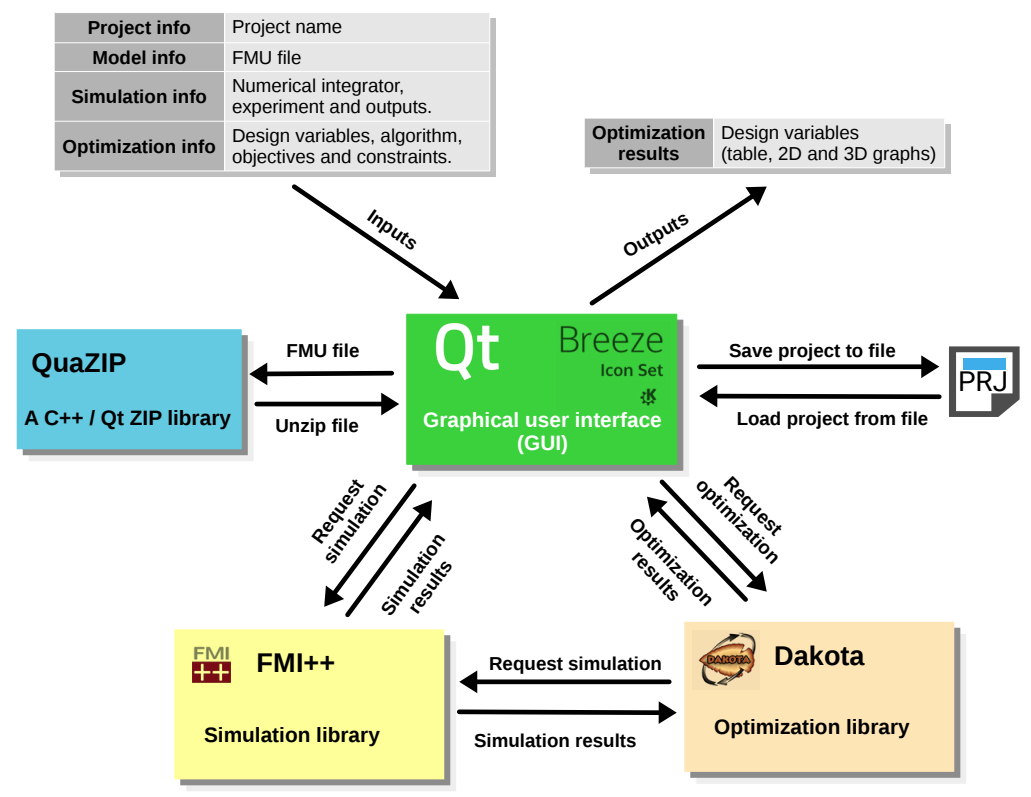

Figure 1. Opt ifmus information exchange

\subsection{State of the art}

Most commercial Modelica tools have parameter estimation and optimization libraries for Modelica models, i.e. Model Design Tools (Elmqvist et al., 2005; Pfeiffer, 2012) in Dymola (Dassault Systemes, 2016) and corresponding libraries in SimulationX (ESI ITI GmbH, 2016), MapleSim (MapleSoft, 2016) and SystemModeler (Wolfram, 2016), among others. GenOpt (Wetter, 2001) is an optimization program that can be coupled with Modelica models compiled in Dymola. BuildingsPy (Berkeley Lab, 2016) is a Python package that can run Modelica simulations using Dymola, additional Python packages for parameter estimation can be used by means of scripting, such as those from SciPy.org (SciPy developers, 2017). The OpenModelica tool (OSMC, 2016) includes the OMOptim tool (Thieriot et al., 2011) for parameter estimation of Modelica models in Windows platforms.

Modelica models can be also exported as FMUs and imported in commercial and open source numerical computational tools such as Matlab/Simulink and Scilab (Scilab Enterprises, 2015). Parameter estimation studies can be performed by means of scripting in these tools. JModelica.org (Åkesson et al., 2010) supports parameter estimation of Modelica and FMI models also by scripting. The RaPId Parameter Identification (RaPId) toolbox (Vanfretti et al., 2016) is a modular and extensible toolbox for parameter estimation of FMI models in Matlab/Simulink.

\section{Optifmus Software Tool}

The under development software tool is called Optifmus. Although it is at an early development stage, it is functional with respect to FMU simulations and parameter estimation studies using a MOGA. The tool is composed of the following main elements.
- Graphical User Interface (GUI). The GUI allows the user to provide all the required information: model information (FMU file and parameters), simulation information (numerical solver and its configuration, inputs and simulation interval), optimization information (algorithm and its configuration, parameters, objective functions and constraints). The GUI also shows the obtained results. Results are presented in tables, 2D and 3D graphs.

- Optimization toolkit. This toolkit collects all the information introduced in the GUI and calls the FMU simulator to perform the needed simulation runs and carry the optimization out using the selected algorithm. Optimization results are presented to the user in the GUI.

- FMU simulator. The simulator performs the model simulation according to the suplied data (model and simulation information) and provides the results to the GUI or to the optimization toolkit.

\subsection{Software Architecture}

Optifmus is being developed in C++ using open source multi-platform libraries and tools. The following list briefly describes the main libraries. Figure 1 shows the information exchange between them.

- Qt toolkit (The Qt Company, 2016). It is a crossplatform application framework used for developing application software. The Qt Core and Qt Widgets modules are used for the GUI. The Qt Charts module is used for 2D graphs, whereas the Qt Data Visualization module is used for 3D graphs.

- Breeze icons (KDE Community, 2016). The GUI icons belong to this open source library. 
- FMI++ (Widl et al., 2013). The FMI++ library is a high-level utility package for FMI-based software development. It provides high-level features, which ease the handling and manipulation of FMU models: an eXtensible Markup Language (XML) parser and numerical integration capabilities. The FMI++ library relies on the Odeint library (Ahnert and Mulansky, 2011), and optionally on SUite of Nonlinear and DIfferential/ALgebraic Equation Solvers (SUNDIALS) (Hindmarsh et al., 2005), for the numerical integration of FMUs.

- QuaZIP (Tachenov, 2016). It is a C++ wrapper for accessing ZIP files. This wrapper uses the Qt toolkit and therefore it is a multi-platform wrapper. It is used in Opt ifmus to handle the extraction of FMU files.

- Dakota (Adams et al., 2016). The Dakota toolkit is intended as a flexible, extensible interface between simulation codes and a variety of iterative systems analysis methods. Dakota is a powerful toolkit which provides the following functionality: optimization, uncertainty quantification, nonlinear least squares methods, and sensitivity/variance analysis. Dakota uses Sandia-developed libraries, as well as external optimization and design of experiments libraries. For further details consult Sandia Corporation (2016). Dakota can be used as a standalone application or as a $\mathrm{C}++$ library.

Additionally, reading and writing operations of input and result files in Comma-separated Values (CSV) or trajectory mat format are performed by means of $\mathrm{C}$ functions available in the source code of the OpenModelica tool.

\section{Examples}

In order to show the optifmus capabilities and illustrate how a parameter estimation study can be performed, the following sections introduce two examples. Section 3.1 shows a steady-state parameter optimization study, whereas Section 3.2 shows a model calibration study against experimental measurements from a real facility.

\subsection{Parameter optimization}

The general formulation for a optimization problem description is given by Equation 1. It can be formulated as optimize (minimize or maximize) several objective functions $f(x)$ that depend on some parameters or design variables $x$ subject to several constraints: upper and lower bounds for design variables, $x_{l}$ and $x_{u}$, equality constraints, $g(x)$ and inequality constraints, $h(x)$.

$$
\begin{array}{ll}
\text { optimize } & f(x) \\
\text { with respect to } & x \in \mathbb{R}^{j} \\
\text { subject to } & x_{l} \leq x \leq x_{u}, \\
& g(x)=0, \\
& h(x) \leq 0,
\end{array}
$$

where,

$$
\begin{aligned}
f(x) & =\left\{f_{1}(x) \cdots f_{i}(x)\right\}, \\
x & =\left\{x_{1} \cdots x_{j}\right\}, \\
x_{l} & =\left\{x_{l, 1} \cdots x_{l, j}\right\}, \\
x_{u} & =\left\{x_{u, 1} \cdots x_{u, j}\right\}, \\
g(x) & =\left\{g_{1}(x) \cdots g_{k}(x)\right\}, \\
h(x) & =\left\{h_{1}(x) \cdots g_{n}(x)\right\} .
\end{aligned}
$$

The example considered in this section, known as Srinivas' problem, can be found in the Dakota User's Manual (Adams et al., 2016), section Additional examples $\rightarrow$ Multiobjective test problems $\rightarrow$ Multiobjective test problem 3 . The problem has two design variables, $x_{1}$ and $x_{2}$ with their respective upper and lower bounds,

$$
\begin{aligned}
& -20 \leq x_{1} \leq 20, \\
& -20 \leq x_{2} \leq 20,
\end{aligned}
$$

two objective functions, $f_{1}$ and $f_{2}$, which must be minimized,

$$
\begin{aligned}
& f_{1}\left(x_{1}, x_{2}\right)=\left(x_{1}-2\right)^{2}+\left(x_{2}-1\right)^{2}+2, \\
& f_{2}\left(x_{1}, x_{2}\right)=9 x_{1}-\left(x_{2}-1\right)^{2},
\end{aligned}
$$

and two inequality constraints $h_{1}$ and $h_{2}$,

$$
\begin{aligned}
& h_{1}\left(x_{1}, x_{2}\right)=x_{1}^{2}+x_{2}^{2}-225 \leq 0, \\
& h_{2}\left(x_{1}, x_{2}\right)=x_{1}-3 x_{2}+10 \leq 0 .
\end{aligned}
$$

The first step is to generate a FMU file of this model, most Modelica tools support exporting Modelica models to FMUs. The Modelica code of this model is as follows.

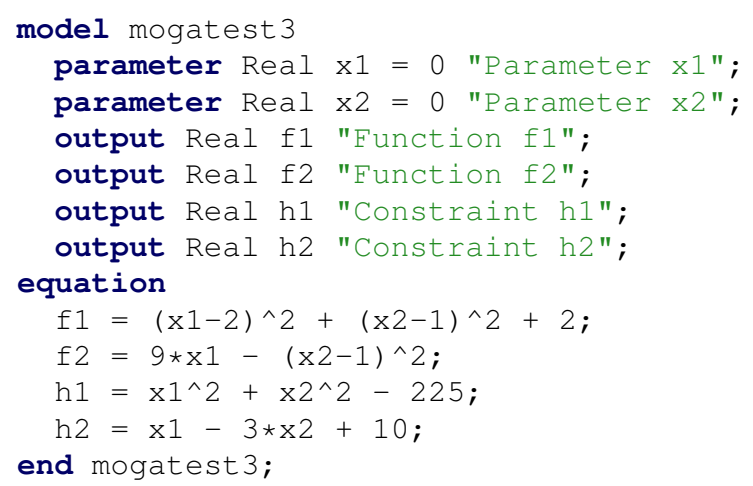

The next step is to create a new project in Optifmus. Currently, two kinds of projects can be created: simulation and parameter estimation. Projects can be saved to and loaded from files. Figure 2 shows the opt ifmus GUI for parameter estimation. The information that must be completed is divided in groups in the GUI and it is described as follows.

1. Project information. A descriptive name can be given to easily identify the project.

2. Model information. A FMU file must be specified. Once the file is loaded, some information is 
Table 1. Numerical integrators

\begin{tabular}{lccc}
\hline Numerical integrator & Library & Step size & Order \\
\hline Forward Euler & Odeint & Constant & 1 \\
$4^{\text {th }}$ order Runge-Kutta & Odeint & Constant & 4 \\
Adams-Bashforth-Moulton & Odeint & Constant & Adjustable \\
$5^{\text {th }}$ order Runge-Kutta-Cash-Karp & Odeint & Controlled & 5 \\
$5^{\text {th }}$ order Runge-Kutta-Dormand-Prince & Odeint & Controlled & 5 \\
$8^{\text {th } \text { order Runge-Kutta-Fehlberg }}$ & Odeint & Controlled & 8 \\
Bulirsch-Stoer & Odeint & Controlled & Controlled \\
$4^{\text {th }}$ Rosenbrock & Odeint & Controlled & 4 \\
Backwards Differentiation Formula (BDF) & SUNDIALS & Controlled & Controlled \\
Adams-Bashforth-Moulton & SUNDIALS & Controlled & Controlled \\
\hline
\end{tabular}

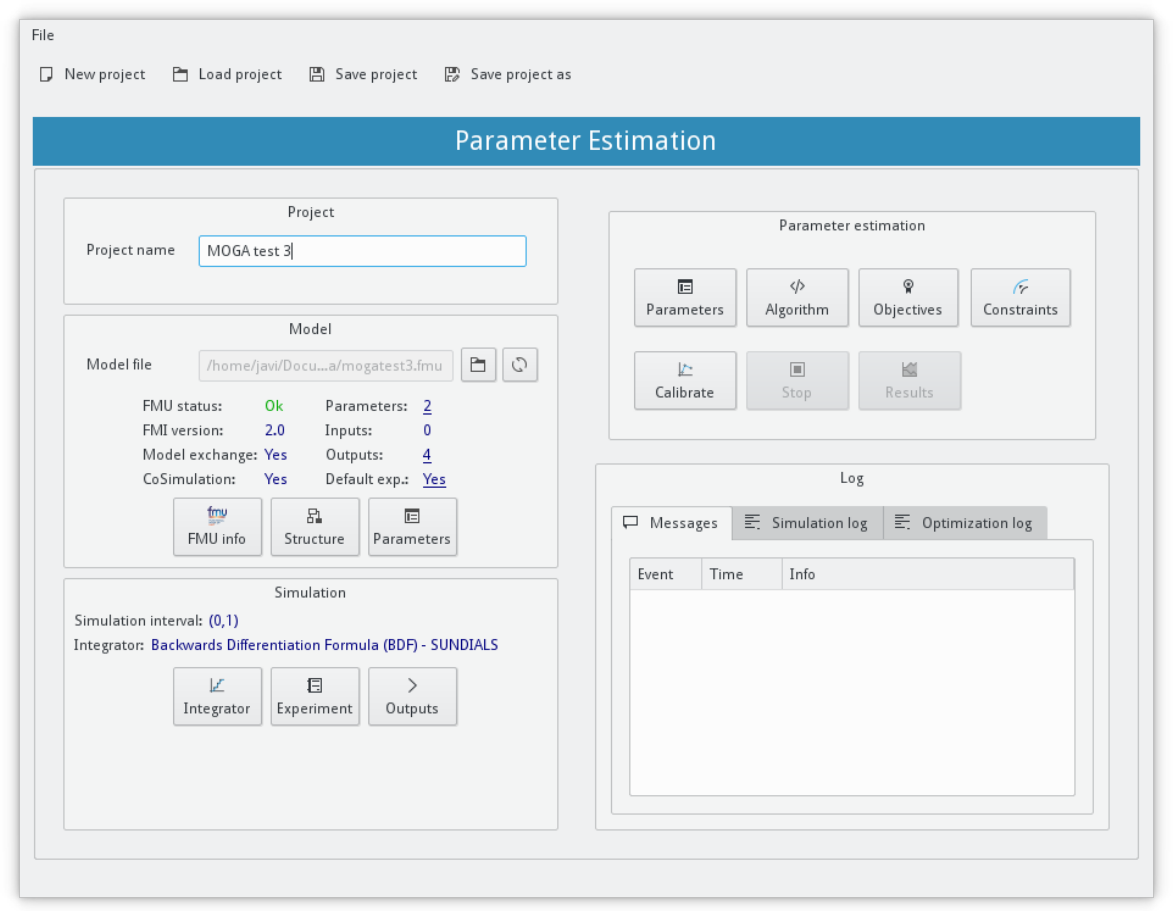

Figure 2. Opt i fmus GUI for parameter estimation.

displayed in the GUI. There are three buttons in this group. The FMU info button shows some information about the model, see Figure $3 \mathrm{a}$. The structure button shows the model structure, see Figure $3 \mathrm{~b}$. The parameters button allows the user to give values to the model parameters, see Figure 3c. Since in our case, both parameters $x_{1}$ and $x_{2}$ are going to be calibrated, there is no need to give them values.

3. Simulation information. There are also three buttons in this group: numerical integrator, experiment and outputs. The first one allows us to select the numerical integrator, Figure 4a. The step size is only needed if it is not controlled by the integrator. If it is controlled, absolute and relative tolerance are used instead. Some integrators allows the users to specify the order whereas others have a fixed constant or a controlled order. The FMI++ library can use the numerical integrators given in Table 1 from the Odeint and SUNDIALS libraries. The numerical integrator (BDF) and tolerances $\left(10^{-4}\right)$ are left by default in our example. The experiment window permits selecting the simulation interval, start and stop times, and matchs model inputs with data from files. Since our model does not have inputs and it is a steadystate model, this step can be omitted and thus leaving the simulation interval by default, $[0,1]$ seconds. Section 3.2 shows how to use this window.

The outputs window, see Figure $4 \mathrm{~b}$, shows the model outputs, furthermore allows us to specify the number of intervals, i.e. the number of points that will be sampled for the output trajectory. The number of points can be also set by a time step instead of by a fixed number. Since our model is a steady-state one, there is no need to sample more than one interval. One interval means that values at the beginning and end of the simulation are stored. 


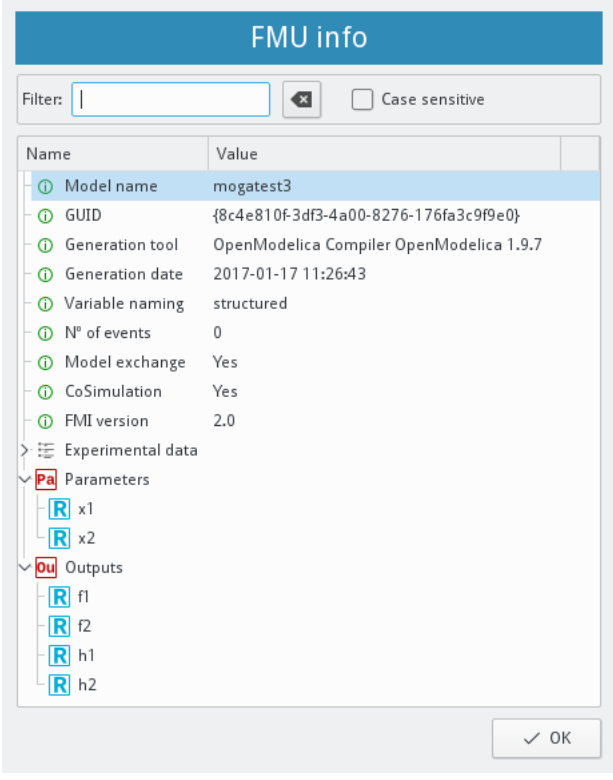

(a) FMU information

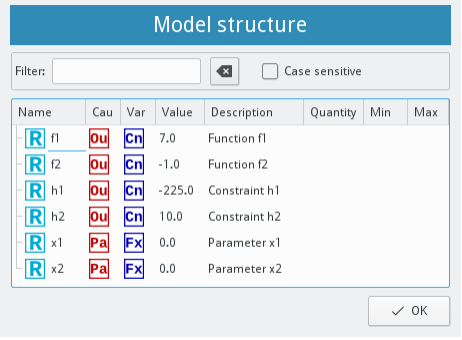

(b) Model structure

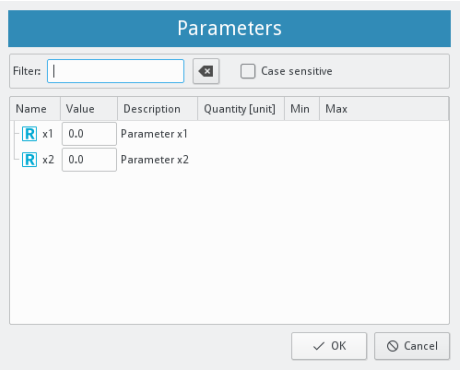

(c) Model parameters

Figure 3. Model information

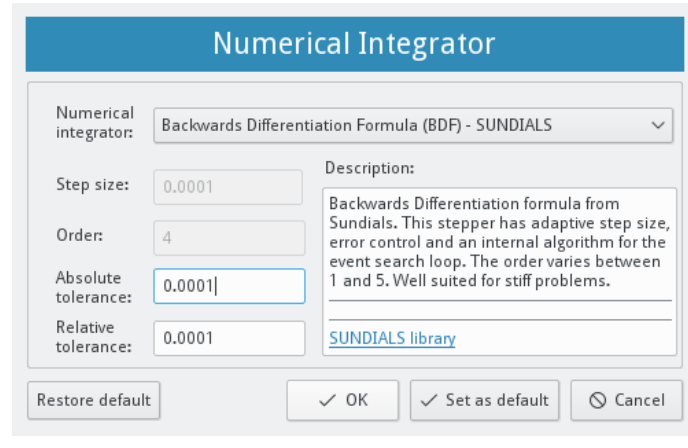

(a) Numerical integrator

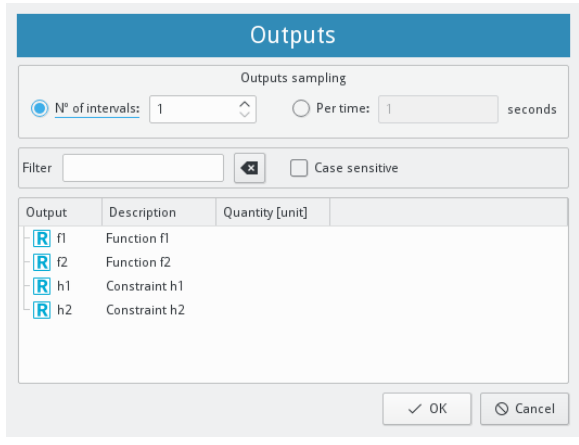

(b) Model outputs

Figure 4. Simulation information

4. Parameter estimation information. This GUI group gathers all the information for the parameter estimation study: parameters or design variables, optimization algorithm, objective functions and constraints.

- Parameters. The parameters to be calibrated can be selected in the parameter window, see Figure 6. For each parameter, it can be specified its initial value, its lower and upper bounds, and if scaling is considered. If this is the case, the scaling type can be a fixed value, logarithm scale or automatic. The two first require a scale value. Consult Dakota documentation for further information about scaling of design variables (Adams et al., 2016).

- Algorithm. Currently, the only Dakota algorithm considered in the tool is the MOGA from the Sandiadeveloped JEGA library (Eddy and Lewis, 2001). This is a multi-objective algorithm which supports general constraints: bounded design variables, linear and nonlinear equality and inequality constraints.
The algorithm is highly configurable. Figure 5 shows the window to configure the algorithm. The options selected in our case are those indicated in Dakota documentation for this example. The number of model evaluations is set to 2000 .

- Objectives. The objectives can be selected in the objectives window, see Figure 7. For each objective the following options are available: criterion (maximize or minimize), trajectory reduction, weight, scaling type and value. The trajectory reduction option reduces the whole trajectory for each objective function to a single value in each simulation run. This is required because the optimization algorithm needs a single value per objective function and simulation run. The following options are available: root mean of squares, mean of absolute values, max, min or last value. The weight value is used if the option to use weights, and therefore transform the multi-objective problem to a single-objective problem, is checked. 


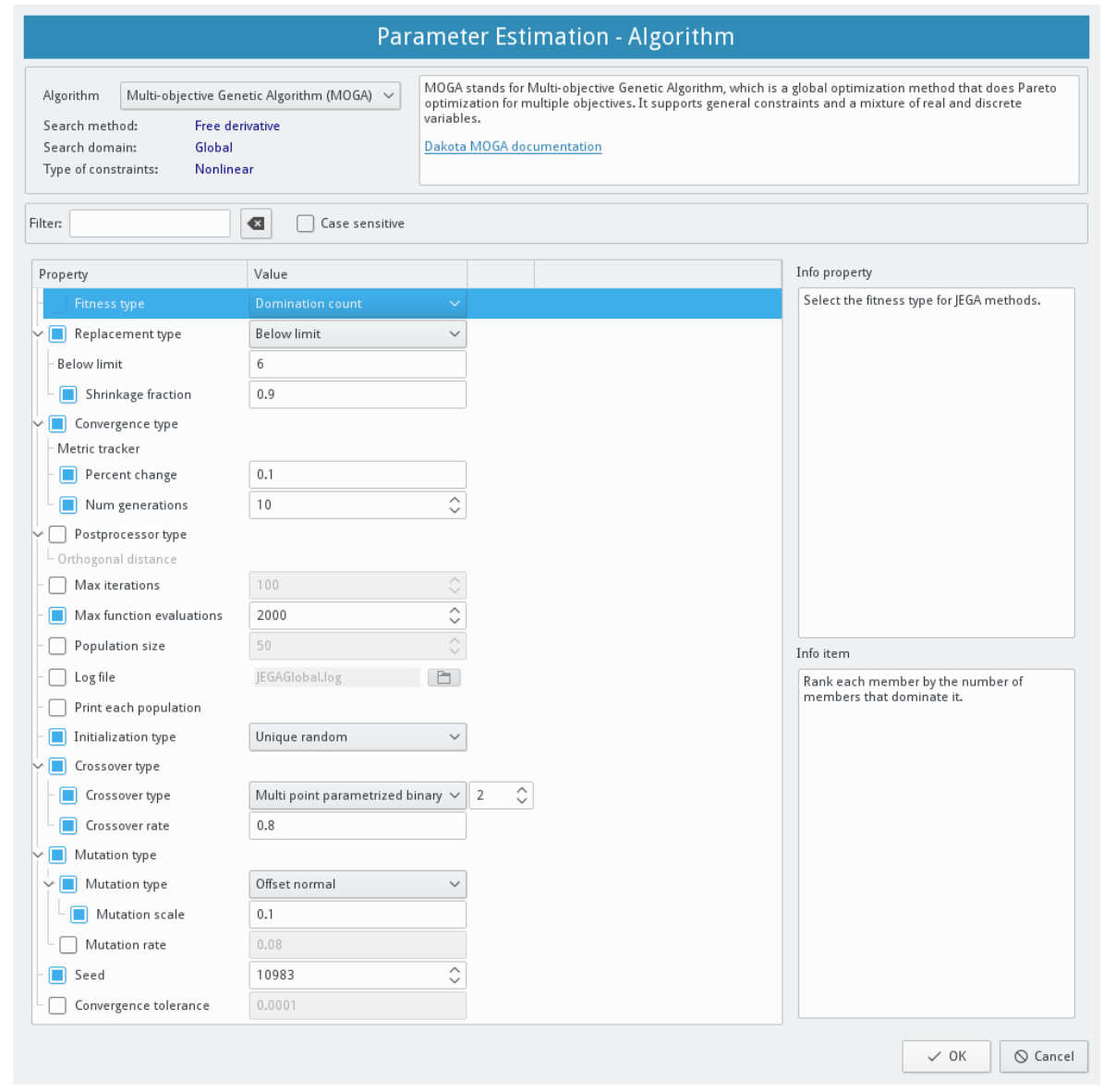

Figure 5. Optimization algorithm configuration

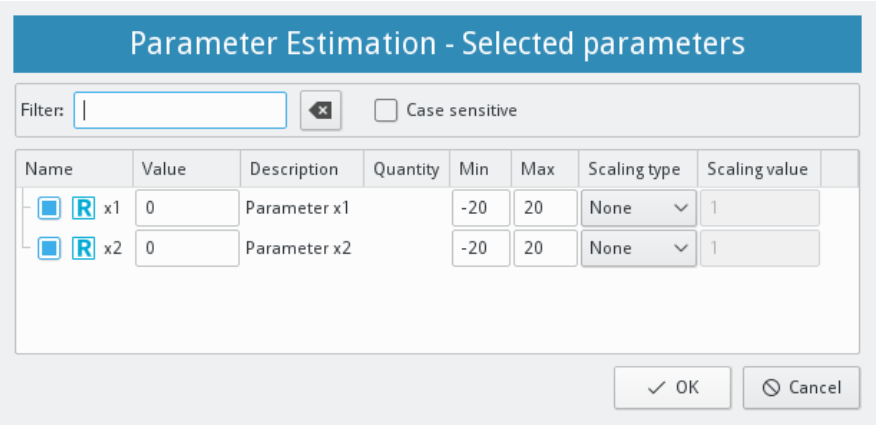

Figure 6. Selected design variables

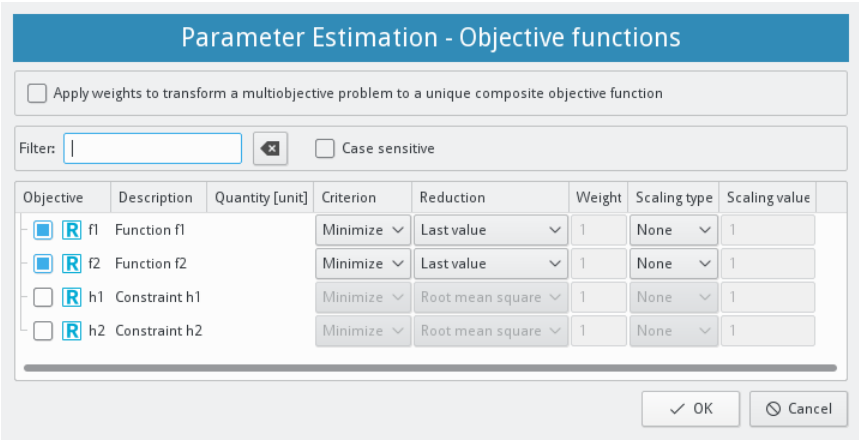

Figure 7. Objective functions

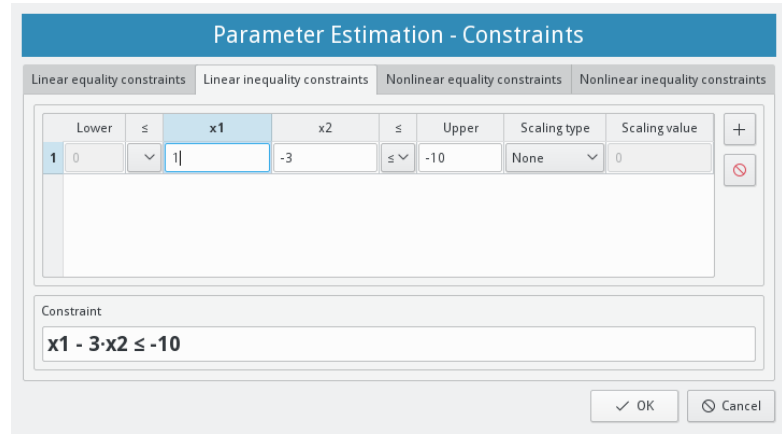

Figure 8. Linear inequality constraints

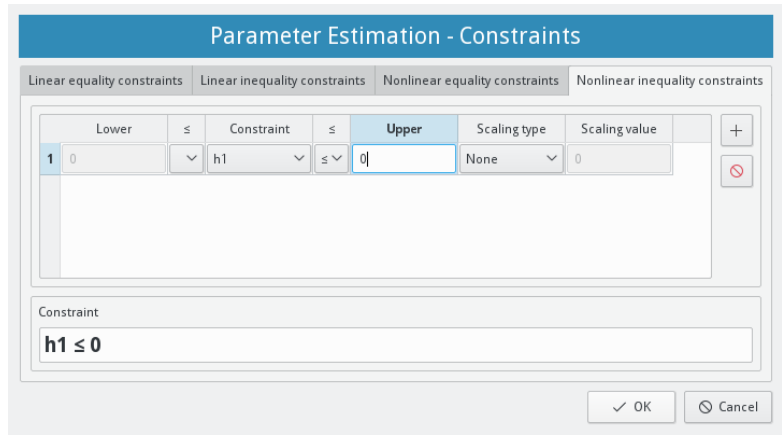

Figure 9. Nonlinear inequality constraints 


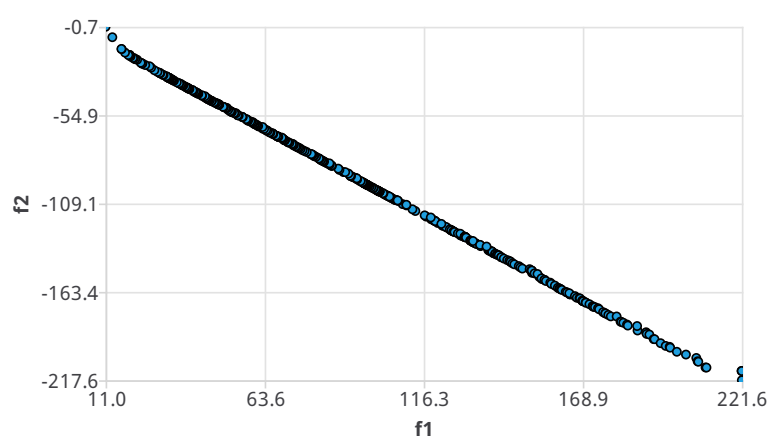

Figure 10. Srinivas' problem - 2D graph

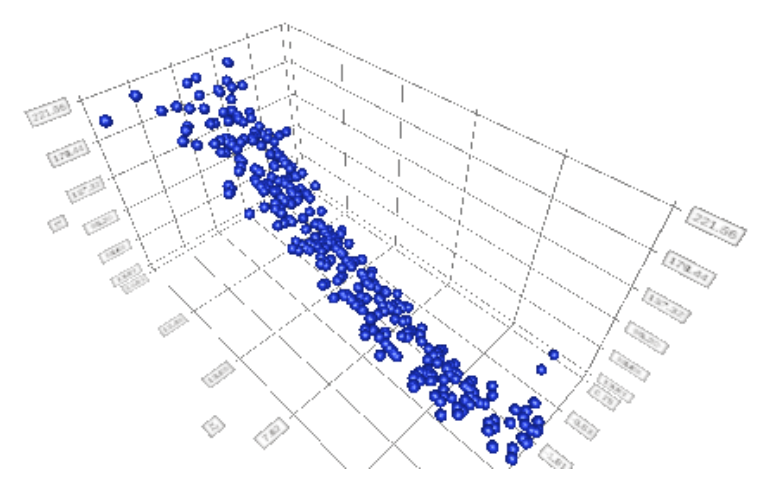

Figure 11. Srinivas' problem - 3D graph

The scaling option has the same meaning than for design variables, but there are some limitations for objective functions, consult Dakota documentation for further details. In our example, for both objective functions the criterion is minimize. Since this is a steady-state model, reductions are set to last simulation values. Scaling is not used and weight are not enabled because this is a multi-objective optimization problem.

- Constraints. The tool supports linear and nonlinear equality and inequality constraints. Scaling options are also available. Linear constraints with respect to design variables can be directly specified in the appropriate tab in the constraint window. In our example, the linear constraint $h_{2}$ was defined in the Modelica code, but this was not necessary since it can be directly defined in the GUI, see Figure 8. On the other hand, it can be also defined in the model as in our example. Nonlinear constraint functions must be defined in the model, in our example $h_{1}$, then both or only one limit per constraint must be set in the GUI, see Figure 9.

Once all previous information is defined, the optimization process can be performed hitting the calibration button, see Figure 2. The stop button allows us to stop the current optimization process. When the calibration process is completed, the results button will be enabled to show

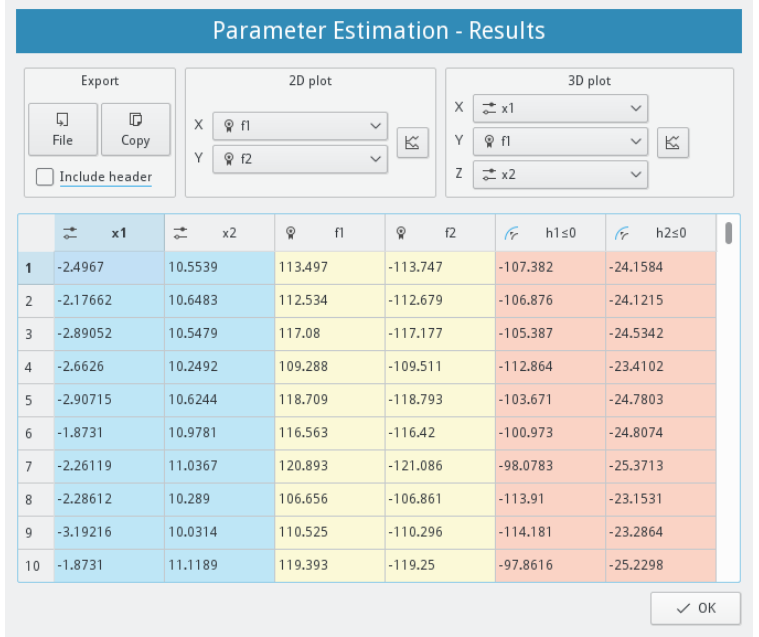

Figure 12. Srinivas' problem - optimization results

information about the optimization results. Our optimization example took less than 5 seconds for 2000 model evaluations in a conventional laptop (4 x Intel Core i5 2.60 $\mathrm{GHz}, 8$ Gbytes of RAM). During the process, messages and $\log$ information are shown in the GUI.

Optimization results are shown in the results window, see Figure 12. The MOGA algorithm provides all the solutions found in or close to the Pareto front. In our case, the MOGA algorithm found 421 solutions after 2000 model evaluations. Design values, objective functions and constraints for each solution are shown in a table. In the result window, any design variable, objective function or constraint can be selected to be plotted in 2D or 3D graphs. Figure 10 shows a $2 \mathrm{D}$ graph of $f_{2}$ with respect to $f_{1}$, which is the Pareto front of our problem. Figure 11 shows a 3D graph of $f_{1}$ with respect to $x_{1}$ and $x_{2}$.

\subsection{Model calibration}

This section presents the calibration of a TES tank dynamic model that it is under development. This kind of tanks is used in solar thermal power plants in order to store thermal energy and dispatch it at night or under unfavorable meteorological conditions. A complete description of the model is out of the scope of this paper, but a brief summary is given in the following lines. The storage fluid is commonly molten salts, which can reach high temperatures.

The dynamic model considers two control volumes and dynamic mass and energy balances for molten salt and the the inert gas in the facility, nitrogen. Tank geometry, slope and dimensions are considered in the model. The pump inside the tank is also modeled, assuming a simplified geometrical form. The position of the level meter and thermocouples in the tank are also taken into account. The model considers different kinds of heat transfer processes: convection, conduction and radiation between molten salt, gas, tank walls, roof, floor, pump, insulation and foundation. The variables of interest are tank level, together with molten salt and gas temperatures. 


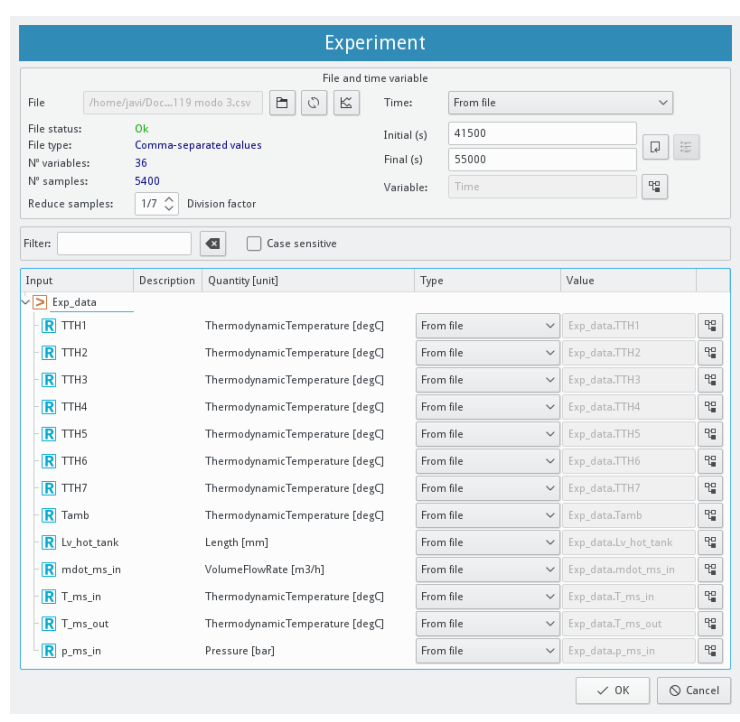

Figure 13. Tank calibration experiment

Molten salt tank foundation designs are commonly outside of standards for foundations, since these standards do not cover the temperature range were TES systems operate. The modeled tank has several foundation layers made of concrete with steel fibers and a compacted light expanded clay aggregate. The thermal insulation is usually made of several layers from different materials. The outer thermal insulation layer is frequently covered with an aluminum jacket for weather protection. For all these reasons, thermal conductivities in the insulation and foundation are difficult values to estimate. In this model, those thermal conductivities are assumed as mean constant values and have been calibrated using the Optifmus tool. Needed measurements are available from experimental campaigns carried out in the facility. It is located at CIEMAT - Plataforma Solar de Almería (PSA). Therefore, our model calibration problem can be formulated as minimizing the differences between molten salt and gas experimental and simulated temperatures by tuning the mean insulation and foundation thermal conductivities.

The steps to perform the calibration are similar to those in Section 3.1, for example setting the project (step 1) and model (step 2) information. The remaining steps are briefly summarized in what follows.

3. Simulation information. The experiment window is used to match model inputs with experimental data stored in files, see Figure 13. The values in the input file can be visualized thanks to a plotting tool included in Optifmus. In case the input file is oversampled, a factor can be used to reduce the number of samples. Model inputs can be also fixed to constant values in this window if needed. Time values can be read from the loaded file or a number of time intervals between the start and stop times can be specified in the GUI. In our example, the simulation interval is set to $[41500,55000]$ seconds. The number of samples is reduced by $1 / 7$ in order to reduce the calibration time, since samples were taken each five seconds. Model inputs and time are matched to file data. The numerical integrator is left by default. The number of intervals is set to 500 in the output window in order to capture several points in the output trajectories.

4. Parameter estimation information. There are no constraints in our example, the remaining configuration options are described as follows.

- Parameters. Insulation and foundation mean thermal conductivities $\left(k_{\text {ins }}, k_{\text {fou }}\right)$ are the design variables, guess initial values are $0.15 \mathrm{~W} /(\mathrm{mK})$. They are bounded in the $[0,1]$ interval.

- Algorithm. The MOGA algorithm is used with its default values, besides the number of model evaluations (1000) and the seed (1) in order to obtain reproducible results.

- Objectives. The differences between experimental and simulated molten salt and gas temperatures are the two objective functions $\left(T_{m s, d i f f}, T_{g a s, d i f f}\right)$, they must be minimized. The trajectory reduction was set to root mean square values, therefore both objective functions provide the mean temperature difference in the trajectory. There is no need to use scaling since both objective functions represent temperature differences. Weights are not used because we are considering a multi-objective minimization problem.

The calibration process took 21 minutes and found 157 solutions in or close to the Pareto front for 1000 model evaluations. Figure 14 shows 10 of those solutions, where the objective functions give the mean temperature difference between experimental data and simulation results. Figure 15 shows the Pareto front.

The first solution in Figure 14, and pointed out by the arrow in Figure 15, was used to compare the model results against a different set of experimental data. All figures shown in this section were created in the Opt i fmus plotting tool. The system was exposed to several mass flow rate steps in this experiment, see Figure 16. Figure 17 shows experimental and simulated tank levels. Horizontal lines point out the position in height of the thermocouples. The gas experimental temperature corresponds to that from the highest-placed thermocouple, whereas the molten salt temperature is obtained from the highestplaced thermocouple immersed in molten salts. Figure 18 shows the experimental and simulated molten salt temperatures. Notice that there is no experimental molten salt temperature until the tank level reaches the first thermocouple position. This is why the experimental temperature is set to a constant value at the beginning of the simulation. Figure 19 shows the experimental and simulated gas temperatures. Notice that the molten salt level reaches the last thermocouple at the end of the simulation, therefore there are no available measurements for gas temperature. 


\begin{tabular}{|c|c|c|c|c|}
\hline & $\vec{a}$ Tank.k_ins & $\vec{a}$ Tank.k_fou & Q Tms_diff & Q Tgas_diff \\
\hline 1 & 0.240195 & 0.465693 & 1.55434 & 2.0533 \\
\hline 2 & 0.240195 & 0.464505 & 1.55488 & 2.05292 \\
\hline 3 & 0.240195 & 0.471824 & 1.55155 & 2.05566 \\
\hline 4 & 0.240195 & 0.473722 & 1.55069 & 2.05635 \\
\hline 5 & 0.240195 & 0.453473 & 1.56001 & 2.04869 \\
\hline 6 & 0.240195 & 0.473362 & 1.55085 & 2.05633 \\
\hline 7 & 0.240195 & 0.451157 & 1.5611 & 2.04785 \\
\hline 8 & 0.240195 & 0.447909 & 1.56263 & 2.04661 \\
\hline 9 & 0.240195 & 0.446876 & 1.56312 & 2.0462 \\
\hline 10 & 0.240195 & 0.440346 & 1.56626 & 2.0438 \\
\hline
\end{tabular}

Figure 14. Tank model calibration results

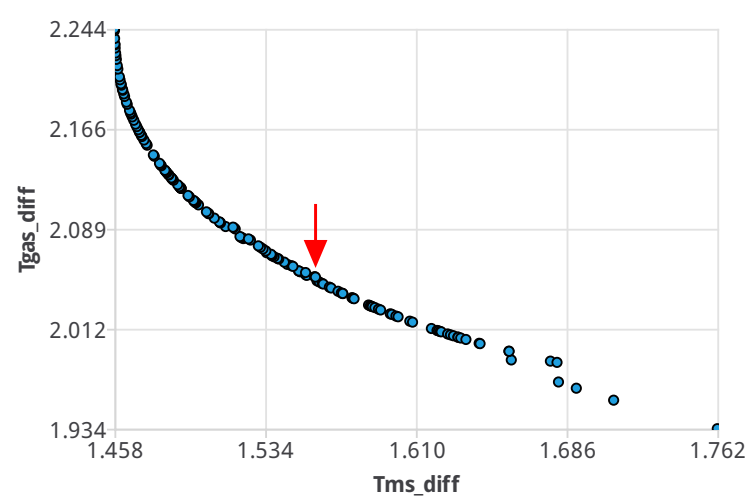

Figure 15. Tank model calibration Pareto front

\section{Optifmus limitations}

The current main Opt ifmus limitations are listed here.

- The software tool has been tested only in Linux. It is planned to be tested in Windows and Mac platforms.

- Only ME FMUs version 1.0 and 2.0 are supported.

- Only continuous real design parameters, objectives functions and constraints are supported.

- All the MOGA options are configurable from the GUI besides the niching type and the use of surrogate models which are not supported.

\section{Ongoing work and future ideas}

Ongoing work is summarized in the following list.

- Optimize the code to improve speed.

- Unit support when setting parameter values.

- Load FMI++ logs in the Opti fmus GUI.

- More graphic configuration options.

- Include an option to simulate the model with the parameters of the selected row in the result table.

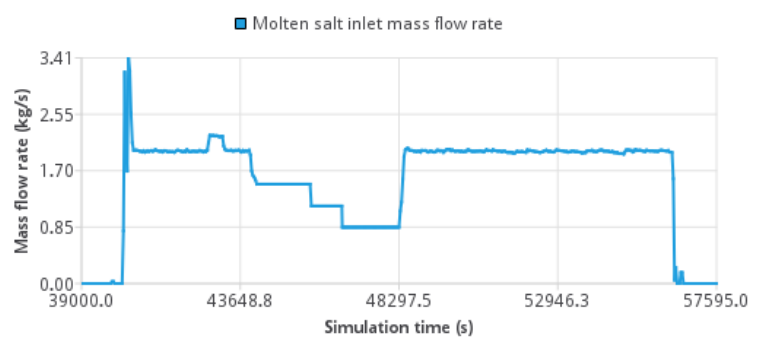

Figure 16. Tank simulation - mass flow rate

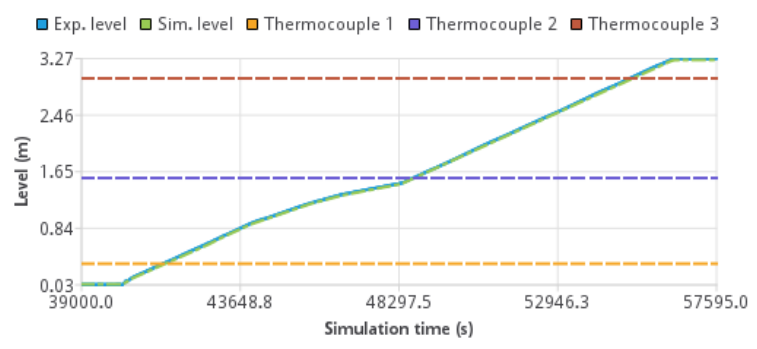

Figure 17. Tank simulation - levels

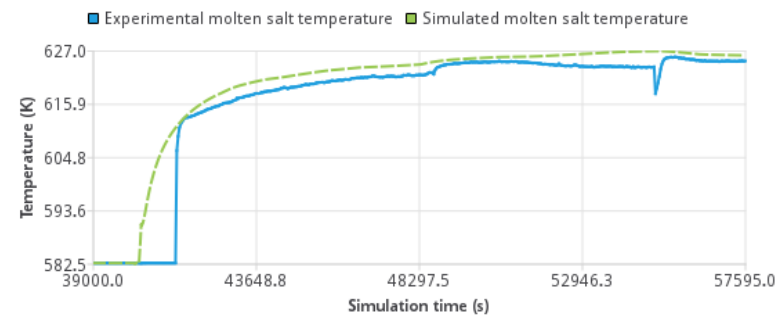

Figure 18. Tank simulation - molten salt temperatures

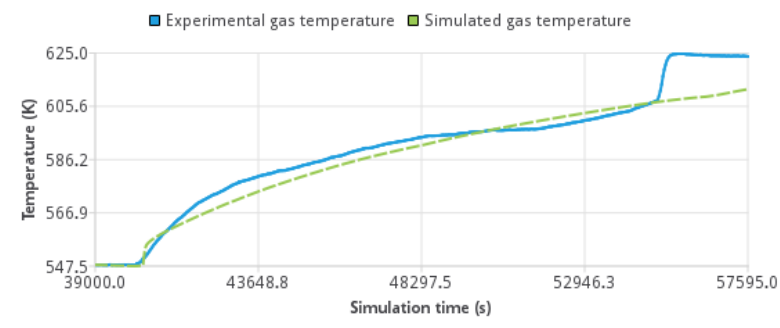

Figure 19. Tank simulation - gas temperatures

- Dakota offers many more algorithms for parameter estimation and optimization. One of the ongoing tasks is to implement several of those algorithms.

As future ideas to improve the software tool, the following will be considered and studied.

- Dakota is a powerful toolkit, other features that could be added to the tool are: parameter studies, sensitivity analyses, design of experiments, uncertainty quantification, and model simplification by means of surrogate models.

- The development of an Application Programming Interface (API) independent of the GUI could be useful for integrating FMI optimization capabilities in other 
tools. It could be applied to offline optimization, as well as to online optimization, for example in MPC.

- Parallelization of the software tool could drastically reduce the optimization time. Dakota offers capabilities for parallelization. If we consider parallelization, as well as an API, executable programs could be generated and executed in high-performance clusters to further reduce the computational time.

\section{Acknowledgments}

This work has been funded by the National Plan Project DPI2014-56364-C2-1/2-R (ENERPRO-EFFERDESAL) of the Spanish Ministry of Economy, Industry and Competitiveness and ERDF funds.

\section{References}

Brian M. Adams, Mohamed S. Ebeida, Michael S. Eldred, Gianluca Geraci, John D. Jakeman, Kathryn A. Maupin, Jason A. Monschke, Laura P. Swiler, J. Adam Stephens, Dena M. Vigil, and Timothy M.Wildey. Dakota, A Multilevel Parallel Object-Oriented Framework for Design Optimization, Parameter Estimation, Uncertainty Quantification, and Sensitivity Analysis: Version 6.5 User's Manual, 2016.

Karsten Ahnert and Mario Mulansky. Odeint - Solving ordinary differential equations in $\mathrm{C}++$. In AIP Conference Proceedings, volume 1389, pages 1586-1589, 2011. ISBN 9780735409569. doi:10.1063/1.3637934.

Johan Åkesson, Karl-Erik Årzén, Magnus Gäfvert, Tove Bergdahl, and Hubertus Tummescheit. Modeling and Optimization with Optimica and JModelica.org-Languages and Tools for Solving Large-Scale Dynamic Optimization Problems. Computers and Chemical Engineering, 34(11):1737$1749,2010$.

Berkeley Lab. BuildingsPy - Modelica Buildings Library, 2016. URL http://simulationresearch.lbl. gov/modelica/buildingspy/.

Dassault Systemes. Dymola 2017 FD01, 2016. URL http: //www.dymola.com.

John Eddy and Kemper Lewis. Effective Generation of Pareto Sets Using Genetic Programming. In ASME 2001 Design Engineering Technical Conferences and Computers and Information in Engineering Conference, number 1, pages 1-9, Pittsburgh, PA, 2001.

Hilding Elmqvist, Hans Olsson, Sven Erik Mattsson, Dag Brück, Christian Schweiger, Dieter Joos, and Martin Otter. Optimization for Design and Parameter Estimation. In Proc. $4^{\text {th }}$ International Modelica Conference, 2005.

ESI ITI GmbH. SimulationX 3.8, 2016. URL http: / / www . simulationx.com/.

Alan C. Hindmarsh, Peter N. Brown, Keith E. Grant, Steven L. Lee, Radu Serban, Dan E. Shumaker, and Carol S. Woodward. SUNDIALS: Suite of Nonlinear and Differential/Algebraic Equation Solvers. ACM Transactions on Mathematical Software, 31(3):363-396, 2005. ISSN 0098-3500. doi:10.1145/1089014.1089020.
KDE Community. Breeze icons, 2016. URL https:// github.com/KDE/breeze-icons.

MapleSoft. MapleSim 2016, 2016. URL https://www. maplesoft.com/products/maplesim/.

Modelica Association. Functional Mock-up Interface for Model Exchange and Co-Simulation, Version 2.0, 2014a. URL https://www. fmi-standard.org/downloads.

Modelica Association. Modelica Specification, version 3.3 Revision 1, 2014b. URL http://www.modelica.org/ documents.

OSMC. OpenModelica 1.9.7, 2016. URL http://www. openmodelica.org/.

Andreas Pfeiffer. Optimization Library for Interactive MultiCriteria Optimization Tasks. In Proc. $9^{\text {th }}$ International Modelica Conference, pages 669-680, Munich, Germany, nov 2012.

Sandia Corporation. Dakota Packages, 2016. URL https: //dakota.sandia.gov/content/packages.

Scilab Enterprises. Scilab: Open Source software for numerical computation, 2015. URL http: //www. scilab.org/.

SciPy developers. SciPy.org - Python-based ecosystem of opensource software for mathematics, science, and engineering, 2017. URL http://scipy.org/.

Sergey A. Tachenov. QuaZIP - Qt/C++ wrapper for ZIP/UNZIP package, 2016. URL http: / / quazip. sourceforge. net/.

The MathWorks Inc. MATLAB R2016b, 2016. URL http: //www.mathworks.es/products/matlab/.

The Qt Company. Qt - Cross-platform software development for embedded \& desktop, 2016. URL https : / / www . qt . io.

Hubert Thieriot, Maroun Nemer, Mohsen Torabzadeh-Tari, Peter Fritzson, Rajiv Singh, and John John Kocherry. Towards Design Optimization with OpenModelica Emphasizing Parameter Optimization with Genetic Algorithms. In Proc. $8^{\text {th }}$ International Modelica Conference, pages 756-762, 2011.

Luigi Vanfretti, Maxime Baudette, Achour Amazouz, Tetiana Bogodorova, Tin Rabuzin, Jan Lavenius, and Francisco José Goméz-López. RaPId: A modular and extensible toolbox for parameter estimation of Modelica and FMI compliant models. SoftwareX, 5:144-149, 2016. ISSN 23527110. doi:10.1016/j.softx.2016.07.004.

Michael Wetter. GenOpt - A Generic Optimization Program. Seventh International IBPSA Conference, (1):601-608, 2001.

Edmund Widl, Wolfgang Muller, Atiyah Elsheikh, Matthias Hortenhuber, and Peter Palensky. The FMI++ library: A high-level utility package for FMI for model exchange. 2013 Workshop on Modeling and Simulation of Cyber-Physical Energy Systems, MSCPES 2013, 2013. doi:10.1109/MSCPES.2013.6623316.

Wolfram. SystemModeler 4.3, 2016. URL http://www . wolfram.com/system-modeler/. 